\title{
In silico Prediction of the Blood-Brain Barrier Permeation: Are We There Yet?
}

\section{Max K Leong ${ }^{1-3 *}$}

${ }^{1}$ Department of Chemistry, National Dong Hwa University, Shoufeng, Hualien 97401, Taiwan

${ }^{2}$ Department of Life Science and Institute of Biotechnology, National Dong Hwa University, Shoufeng, Hualien 97401, Taiwan

${ }^{3}$ Department of Medical Research and Teaching, Mennonite Christian Hospital, Hualien, Hualien, 97059, Taiwan

The drug discovery and development has turned to a new chapter ever since the implementation of the Brain Research through Advancing Innovative Neurotechnologies (BRAIN) in the United States of America and Human Brain Project (HBP) in the European Union. Both initiatives have signified the imminence of finding the treatment for the central nervous system (CNS) disorders such as Alzheimer's disease (AD), Parkinson's disease (PD), brain cancer, and stroke, which, in turn, are substantially affected by aging. Nevertheless, the average lifespan of people increases gradually, resulting in the fact that more and more people are now living with $\mathrm{AD}$, and, more practically, the health care for the CNS diseases will become substantially financial burdens around the globe.

Blood-brain barrier (BBB) is a brain capillary endothelial wall in the circulatory system that prevents many substances from entering the CNS. Actually, the inability to cross the BBB is one of the major hurdles to efficaciously deliver therapeutics to the brain as manifested by the fact only an extremely small portion of small-molecule therapeutic agents and nearly none of macromolecule biopharmaceuticals can cross the BBB. The BBB permeation, which can take places through various routes, including passive diffusion, carrier mediated influx transport, active efflux transport, is one of critical parameters should be taken into account in the process of drug discovery and development to reduce CNS-related adverse side-effects for systemically targeted drug candidates or to design CNS-targeted molecules that can cross the BBB.

In addition to a variety of in vivo, ex vivo, and in vitro assay systems, in silico approaches have played an increasingly important role to assess the BBB permeation. Indeed, numerous quantitative and qualitative structure-activity relationship (QSAR) models have been reported. Nevertheless, it is not uncommon to find that some quantitative models were developed based on assay data using different animal species, such as rats and mice or some models included different data types, such as brain-to-plasma partition coefficient of the bound compound $(K \mathrm{p})$ and that of the free compound (Kp,uu). Those factors constitute various levels of heterogeneity in the sample data, hampering the integrity of developed models consequently.

Furthermore, there is no guarantee that a good predictive model can be derived even based on the highly homogeneous sample data.
For instance, most of linear quantitative models exhibited a linear relationship between hydrophobicity $(\log P)$ and $\log K \mathrm{p}$ value that is not completely correct since a more hydrophobic molecule can more easily cross the $\mathrm{BBB}$, whereas it can stay trapped in the membrane once it is too hydrophobic as illustrated by the fact that the marketed CNS drugs have an optimal range of hydrophobicity. As such, substantial deviations can be expected when a predictive model, which is derived based on more lipophilic samples, is applied to those more hydrophobic compounds or vice versa. In addition to hydrophobicity, more descriptors, which correlate with the BBB permeation nonlinearly, can be expected. Accordingly, those linear models cannot be completely applicable to all molecules.

Conversely, machine learning schemes seemingly provide better alternatives to develop in silico models to predict the BBB permeation since mathematically they can handle such nonlinear relationship. Machine learning schemes, nevertheless, are not the total solution to all questions mainly because of complex nature of the $\mathrm{BBB}$ permeation, leading to the fact that different BBB permeation routes may require different selections of descriptors or even the same descriptor may correlate with different permeation routes in different ways. For instance, the descriptors required to describe the efflux transport by p-glycoprotein are different from those used to depict the influx transport by organic cation transporter 1 . Such discrepancies in the permeation routes can be possibly resolved once the studied compounds can be accurately classified provided that such $a$ priori knowledge is available. Challenges still remain unanswered if molecules can cross the $\mathrm{BBB}$ via more than one route, leading to inappropriate classifications consequently or when the predictive models were to be applied to novel molecules, which actually do not exist or whose permeation routes are completely unknown.

To this end, all published models cannot comprehensively take into consideration all the factors associated with the complex process of BBB permeation. It is impractical to expect a true model can be built. As George Box stated that "all models are wrong-but some models are useful." More advanced and sophisticated algorithms or schemes are much needed to develop genuinely useful models to predict the BBB permeation. Until then, there is still a long and winding route ahead of us.

${ }^{*}$ Corresponding author: Max K. Leong, Department of Medical Research and Teaching, Mennonite Christian Hospital, Hualien, Hualien-97059, Taiwan, Tel: +886 3863 3609; E-mail: leong@mail.ndhu.edu.tw

Received January 20, 2015; Accepted March 24, 2015; Published March 26 2015

Citation: Leong MK (2015) In silico Prediction of the Blood-Brain Barrier Permeation: Are We There Yet? Med chem 5: 130-130. doi:10.4172/21610444.1000254

Copyright: (C) 2015 Leong MK. This is an open-access article distributed under the terms of the Creative Commons Attribution License, which permits unrestricted use, distribution, and reproduction in any medium, provided the original author and source are credited. 